\title{
Photostimulated Luminescence and Thermoluminescence of LSO Scintillators
}

\author{
R. Visser, C. L. Melcher, and J. S. Schweitzer \\ Schlumberger-Doll Research, Old Quarry Rd., Ridgefield, CT 06877-4108 \\ H. Suzuki and T.A. Tombrello \\ California Institute of Technology, Pasadena, CA 91125
}

\begin{abstract}
Photostimulated luminescence (PSL) and thermoluminescence (TL) from five $\mathrm{Lu}_{2(1-x)} \mathrm{Ce}_{2 \times}\left(\mathrm{SiO}_{4}\right) \mathrm{O}$ (LSO) crystals with different light outputs is reported. Optical irradiation into the $\mathrm{Ce}^{3+}$ absorption bands causes the appearance of a broad absorption band near $280 \mathrm{~nm}$ which is ascribed to $\mathrm{Ce}^{4+}$. In addition, a tail is observed extending beyond $700 \mathrm{~nm}$. Optical irradiation into this tail (PSL) or heating of the crystal (TL) results in $\mathrm{Ce}^{3+}$ emission. It is shown that both PSL and TL are due to the same traps. In addition, an anti-correlation is found between the light output under gamma-ray irradiation and the trap concentration in the crystal. The nature of the recombination centers responsible for the low light output in some crystals is not clear. Annealing experiments suggest that the traps and the recombination centers may be related to oxygen vacancies.
\end{abstract}

\section{INTRODUCTION}

$\mathrm{Lu}_{2(1-\mathrm{x})} \mathrm{Ce}_{2 \mathrm{x}}\left(\mathrm{SiO}_{4}\right) \mathrm{O}$ (LSO) is known as a fast and intense scintillator. The decay of the scintillation pulse is almost a single exponential with a decay time of about $40 \mathrm{~ns}$ and the light output can be as high as 28000 phot/MeV $(75 \%$ of $\mathrm{NaI}(\mathrm{Tl})$ ) [1]. The scintillation decay time is close to the decay times of the two $\mathrm{Ce}^{3+}$ centers $(\mathrm{Ce} 1$ and $\mathrm{Ce} 2)$ present in the crystal [2]. This shows that the $\mathrm{Ce}^{3+}$ ions are excited on a very short time scale after the capture of a radiation quantum and that energy transfer from excited $\mathrm{Ce}^{3+}$ ions to quenching centers is negligible.

In our laboratory, we have grown several $\mathrm{Lu}_{2(1}$. ${ }_{x} \mathrm{Ce}_{2 \mathrm{x}}\left(\mathrm{SiO}_{4}\right) \mathrm{O}$ crystals. For the best crystals, the previous paragraph applies. But for some crystals, the light output is considerably lower than the value of 28000 phot $/ \mathrm{MeV}$. This can not be explained by a lower $\mathrm{Ce}^{3+}$ emission efficiency for two reasons: 1) in optical excitation experiments we have found that the $\mathrm{Ce}^{3+}$ emission intensity per photon absorbed by $\mathrm{Ce}^{3+}$ is about the same for all crystals and 2) the scintillation decay time in low light output crystals is only $25 \%$ smaller than in high light output crystals. Thus, the low light output in some crystals is due to a poor energy transfer from free electrons and holes to $\mathrm{Ce}^{3+}$. It is noteworthy that undoped $\mathrm{Lu}_{2}\left(\mathrm{SiO}_{4}\right) \mathrm{O}$ has only very weak emission, even at $11 \mathrm{~K}$, which suggests that in $\mathrm{Lu}_{2(1-x)} \mathrm{Ce}_{2 x}\left(\mathrm{SiO}_{4}\right) \mathrm{O}$, the $\mathrm{Ce}^{3+}$ ions are excited directly by electrons and holes and not by some intermediate center intrinsic to the host material. Then, the light output of the LSO crystal is determined by the amount of electrons and holes that can reach the $\mathrm{Ce}^{3+}$ ions before being trapped or annihilated at recombination centers.

In order to obtain some insight into the nature of the traps and recombination centers present in the LSO crystals, we investigated samples from four crystal boules with different light outputs. It is known that ionizing radiation or UV radiation fills traps that can be subsequently observed in a thermoluminescence (TL) experiment [3]. For this reason, we investigated the TL of our samples. In addition, we performed photostimulated luminescence (PSL) measurements in order to relate the $\mathrm{TL}$ to the optical properties of our samples.

\section{EXPERIMENTAL}

$\mathrm{Lu}_{2(1-x)} \mathrm{Ce}_{2 x}\left(\mathrm{SiO}_{4}\right) \mathrm{O}$ crystals were grown by the Czochralski technique. The raw materials were $99.99 \%$ pure $\mathrm{Lu}_{2} \mathrm{O}_{3}, \mathrm{SiO}_{2}$, and $\mathrm{CeO}_{2}$. After mixing and pressing the raw materials into pellets, they were melted in an iridium crucible which was inductively heated. The furnace assembly was located in a sealed chamber and crystal growth was carried out under a continuous flow of $\mathrm{N}_{2}+3000 \mathrm{ppm} \mathrm{O}_{2}$. As grown, the crystal boules were typically $\sim 20 \mathrm{~mm}$ in diameter and 40 - $60 \mathrm{~mm}$ long.

We denote the four crystal boules investigated in this study by $A, B, C$, and D, where $A$ has the highest light output, B the next highest, etc. The Ce concentration was measured by ICP mass spectrometry by $\mathrm{X}$-ray Assay Laboratories, Don Mills, Ontario: $x=0.00066$ (A), 0.0021 (B), 0.0014 (C) and $0.00064(D)$. Small samples were taken from the boules. For optical absorption measurements polished samples were taken from each of the boules. For thermoluminescence measurements four unpolished chips of about $3 \times 3 \times 1 \mathrm{~mm}^{3}$ were taken. From boule $A$, which turned out to be inhomogeneous, an additional unpolished $3 \times 3 \times 1 \mathrm{~mm}^{3}$ sample was taken. Optical absorption measurements were carried out using a Hitachi U-3210 spectrophotometer. Thermoluminescence measurements were carried out using the Harshaw model 2000-A thermoluminescence detector and the 


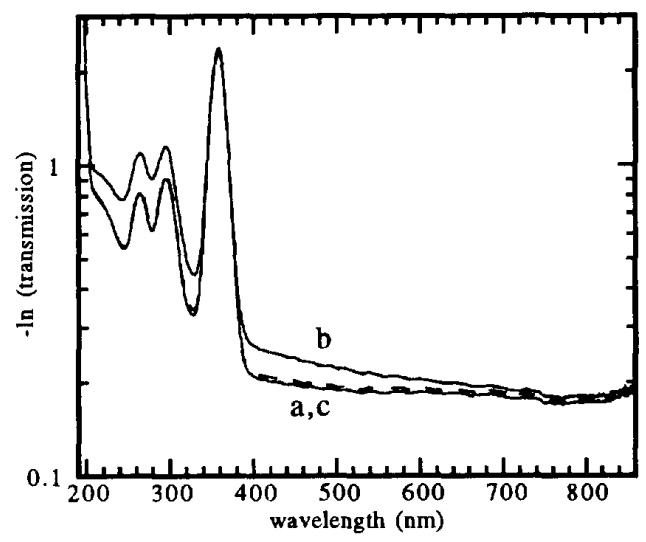

Figure 1. Optical absorption spectrum of a $0.49 \mathrm{~mm}$ thick sample from boule $\mathrm{D}$. Curve a shows the spectrum after heating the sample to $460{ }^{\circ} \mathrm{C}$. Curve $\mathrm{b}$ is taken after an additional 8 minutes irradiation with a $\mathrm{Hg}$ lamp (surface irradiation dose $-10^{18}$ photons $/ \mathrm{cm}^{2}$, with wavelength mainly $<350 \mathrm{~nm}$ ). Curve $c$ (dashed) is measured after an additional 11 minute irradiation at wavelengths $>500 \mathrm{~nm}$ (surface irradiation dose $-10^{19}$ photons $/ \mathrm{cm}^{2}$ ).

model 2000-B automatic integrating picoammeter coupled to a personal computer through a Fluke $8840 \mathrm{~A}$ digital multimeter. Optical excitation experiments were carried out using a Spex Fluorolog-2 spectrofluorometer. The exciting beam was incident perpendicular on the sample surface; emission was recorded at $22.5^{\circ}$ relative to the exciting beam. All spectra were corrected for the equipment's detection efficiency. For annealing the unpolished $3 \times 3 \times 1 \mathrm{~mm}^{3}$ samples were placed on a larger LSO piece resting on Ir wires in an alumina boat that was placed in a programmable Lindberg furnace.

\section{RESULTS}

We performed optical and TL measurements on the samples from the four boules. First, PSL and other optical properties of these samples are presented. Next, TL measurements are discussed.

\section{A. PSL and other optical properties}

In figure 1, the optical absorption spectrum of a polished sample from boule $D$ is shown. Curve a is measured after heating the sample to $460^{\circ} \mathrm{C}$, thereby emptying several traps and eliminating afterglow. At 357,294, 263 and $216 \mathrm{~nm}$ we observe $\mathrm{Ce}^{3+}$ absorption bands. At wavelengths shorter than $200 \mathrm{~nm}$ the absorption edge of the host lattice is observed. Curve $b$ is taken after irradiation with a $\mathrm{Hg}$ lamp, which mainly emits at wavelengths where $\mathrm{Ce}^{3+}$ absorption is prominent. Clearly, this irradiation causes additional absorption features. After the measurement of curve $b$, the

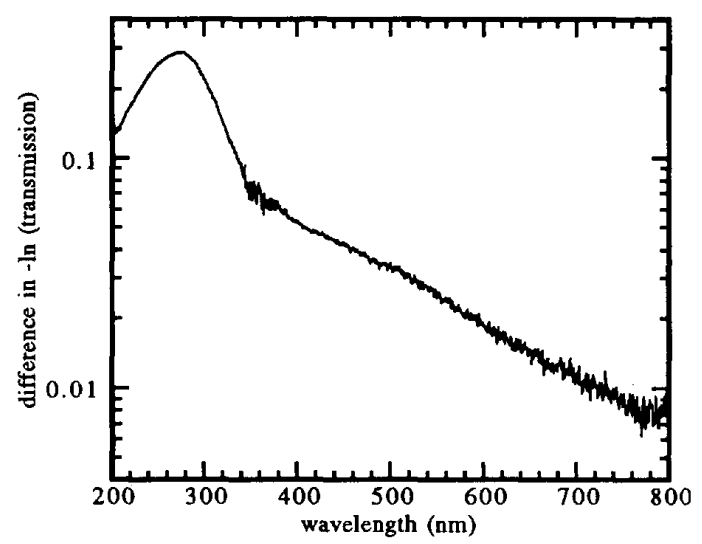

Figure 2. The difference between the absorption curves $b$ and $a$ in figure 1.

sample was irradiated with a W lamp having a $500 \mathrm{~nm}$ cut-off filter. As evident in figure 1, this treatment restored the original absorption spectrum.

In figure 2 , we show the difference between curves $b$ and $a$ in figure 1 . This difference is the absorption induced by the $\mathrm{Hg}$ lamp irradiation. Figure 2 shows a broad absorption band peaking at $276 \mathrm{~nm}\left(36 \cdot 10^{3} \mathrm{~cm}^{-1}\right)$ which has a FWHM width of $105 \mathrm{~nm}\left(15 \cdot 10^{3} \mathrm{~cm}^{-1}\right)$. Spectra similar to those shown in figure 1 and 2 were obtained for polished samples from the other boules, but the magnitude of the difference spectra is smaller for these samples.

Figure 3 shows the excitation spectrum of a polished sample from boule $\mathrm{A}$, which was irradiated with the $\mathrm{Hg}$ lamp. The emission from $\mathrm{Ce}^{3+}$ is recorded at $428 \mathrm{~nm}$ wavelength. The excitation spectrum resembles the absorption spectra of figure 1. At wavelengths shorter than $400 \mathrm{~nm} \mathrm{Ce}^{3+}$ excitation bands are visible, whereas at wavelengths longer than $400 \mathrm{~nm}$ a "tail" is observed extending beyond $700 \mathrm{~nm}$. The PSL. spectrum, obtained when exciting into this tail using 504.5 $\mathrm{nm}$ wavelength light, is shown in figure 4 (curve b).

Also in figure 4 is the afterglow spectrum, recorded when using no exciting light and taken prior to the PSL spectrum (curve a). The shape of both spectra is almost identical, a small difference being due to decay of the emission intensity during the measurement of curve $b$ (from short to long wavelengths). Results on samples from boules $B, C$ and $D$ are similar. The spectral shape differed somewhat from boule to boule (indicating different relative emission intensities due to $\mathrm{Ce} 1$ and $\mathrm{Ce} 2$ centers-see [2]), but the spectral shape of the afterglow was always almost identical to that of the PSL.

The above shows that $\mathrm{Hg}$ lamp irradiation induces the absorption features shown in figure 2 and the excitation properties related to it, whereas light with a wavelength larger than $500 \mathrm{~nm}$ reduces this absorption. This suggests that the 


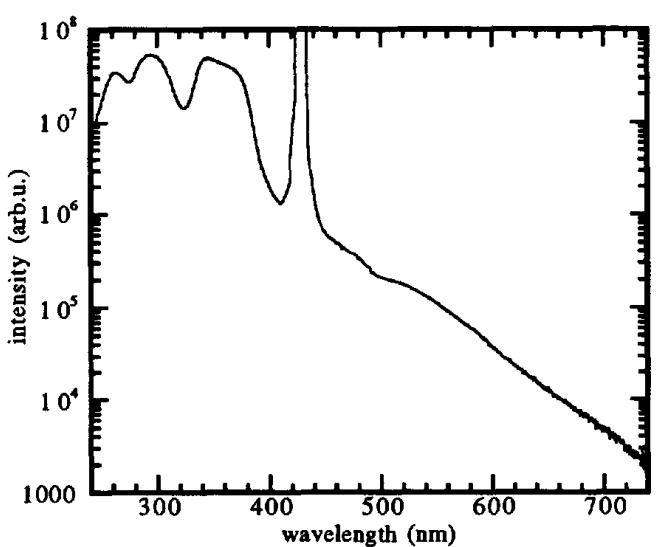

Figure 3. Excitation spectrum of a $1.57 \mathrm{~mm}$ thick polished sample from boule $A$ after 3 minutes irradiation with the $\mathrm{Hg}$ lamp (accumulated surface dose is $\sim 10^{18}$ photons $/ \mathrm{cm}^{2}$ ). The emission wavelength is $428 \mathrm{~nm}$. In order to eliminate second order features, beyond $450 \mathrm{~nm}$ a cut-off filter was used.

absorption features in figure 2 are caused by $\mathrm{Ce}^{3+}$ excitation. In order to prove this, for an unpolished $3 \times 3 \times 1 \mathrm{~mm}^{3}$ sample from boule $\mathrm{A}$, we first bleached the absorption feature by irradiating the crystal with $504.5 \mathrm{~nm}$ light. Next, during several seconds we irradiated the crystal with monochromatic light (resolution: $3 \mathrm{~nm}$ FWHM). The accumulated surface light dose was $\sim 10^{14}$ to $\sim 10^{16}$ photons $/ \mathrm{cm}^{2}$, depending on the wavelength used. After this treatment, we excited the crystal with $504.5 \mathrm{~nm}$ light of a fixed intensity and measured the intensity of the resulting $\mathrm{Ce}^{3+}$ emission (PSL) at $400 \mathrm{~nm}$. The PSL intensity was divided by the accumulated surface light dose. We call the quantity obtained in this way, the PSL creation efficiency. The PSL creation efficiency as a function of irradiation wavelength is shown in figure 5 . Also shown in figure 5 is the excitation spectrum of the $\mathrm{Ce}^{3+}$ emission for this crystal. The correspondence of the PSL creation efficiency with the $\mathrm{Ce}^{3+}$ excitation spectrum is clear. This means that the tail in the excitation spectrum (cf figure 3 ) is caused by $\mathrm{Ce}^{3+}$ excitation and the energy storage processes following it. For wavelengths shorter than $340 \mathrm{~nm}$, there is some difference between the two sets of data in figure 5 , indicating that the PSL creation efficiency is also governed by some other process.

\section{B. $T L$ and its relation to $P S L$}

For the five unpolished samples we measured the TL after irradiating with the $\mathrm{Hg}$ lamp for 8 minutes. In order to avoid differences in the irradiated crystal volume from sample to sample because of differences in sample size, during irradiation an aluminum plate with a $1 \mathrm{~mm}$ diameter hole in it was placed on the crystals resulting in the same light dose for each crystal: $\sim 10^{17}$ photons, corresponding to a surface accumulated light dose of $\sim 10^{18}$ photons $/ \mathrm{cm}^{2}$. At this dose, a large fraction

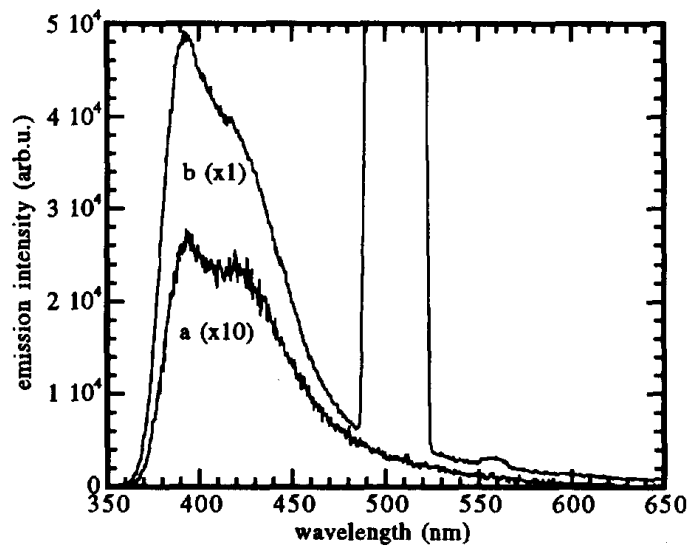

Figure 4. Afterglow spectrum of an unpolished sample from boule A (curve a, multiplied by 10 for clarity). Also shown is the spectrum obtained under the same circumstances, but when additionally exciting the crystal with $504.5 \mathrm{~nm}$ light (curve b). The structure at $560 \mathrm{~nm}$ is an artifact.

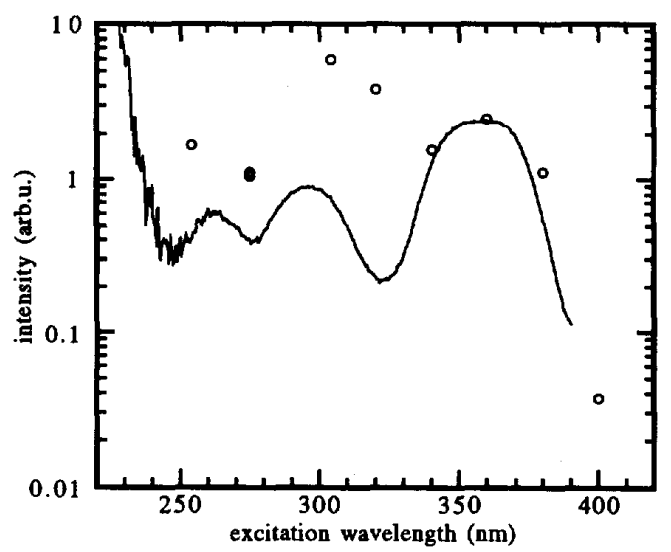

Figure 5. The PSL creation efficiency for the highest light output $3 \times 3 \times 1 \mathrm{~mm}^{3}$ sample from boule $A$ (circles). For comparison, the excitation spectrum recorded at $400 \mathrm{~nm}$ emission wavelength and using the same experimental setup is shown (solid curve).

of the traps responsible for the TL are expected to be filled, because the integrated TL intensity was not much different from that seen after only 3 minutes $\mathrm{Hg}$ lamp irradiation. The TL intensity after 8 minutes was between $2 \%$ (highest light output sample) and $45 \%$ (lowest light output sample) higher than after 3 minutes irradiation.

In figures 6 and 7 the TL spectra obtained after 8 minutes $\mathrm{Hg}$ lamp irradiation are shown for the highest light output unpolished sample from boule $A$ and the unpolished sample from boule $D$, respectively. The spectrum in figure 6 , curve ia is similar to that reported earlier by Dorenbos et al. [3]. The spectrum in figure 7 , curve a, is much broader and more 


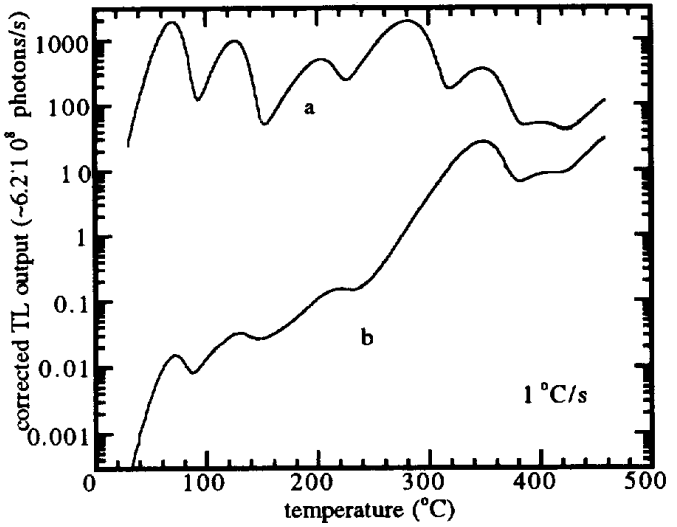

Figure 6. Corrected TL spectra for the highest light output unpolished sample from boule $A$. Curve a shows the TL spectrum measured after 8 minutes irradiation with the $\mathrm{Hg}$ lamp. Curve b shows the TL spectrum measured after 8 minutes irradiation with the $\mathrm{Hg}$ lamp, followed by a 16 minutes bleach using $504.5 \mathrm{~nm}$ light (accumulated surface dose $-10^{19}$ photons $/ \mathrm{cm}^{2}$ ). The heating rate is $1^{\circ} \mathrm{C} / \mathrm{s}$.

intense. The measurement of curves a in the figures was started within a few minutes after the end of the excitation. The TL spectra for samples from boules A and B all look similar to those in figure 6; those for the sample from boule $C$ are similar to those in figure 7. All spectra in the figures 6 and 7 were corrected for the temperature dependence of the $\mathrm{Ce}^{3+}$ emission [4]. This was done by multiplying the measured TL spectrum by the factor $[1+\exp (13-\{4616.3 / T\})]$, where the temperature $\mathrm{T}$ is in Kelvin.

In addition to the TL spectra obtained after irradiation with the $\mathrm{Hg}$ lamp, figures 6 and 7 also show the TL spectra after PSL-bleaching with $504.5 \mathrm{~nm}$ light. During this bleaching process, we recorded the $\mathrm{Ce}^{3+}$ emission intensity at $400 \mathrm{~nm}$. During the PSL-bleaching, the $\mathrm{Ce}^{3+}$ emission intensity decreased by a factor of about 900 for the sample from boule $A$ and 2000 for the sample from boule $D$. The shape of the tail in the excitation spectrum at $400 \mathrm{~nm}$ emission wavelength (cf figure 3) was unchanged by the PSL bleaching at $504.5 \mathrm{~nm}$; only its intensity decreased. As figures 6 and 7 show, after the PSL-bleaching, the TL signal is much lower than before the PSL-bleaching. This means that the majority of the traps giving rise to the $\mathrm{TL}$ also give rise to the PSL and therefore have absorption at $504.5 \mathrm{~nm}$.

We have also measured the PSL after the TL measurements shown by the curves a in figures 6 and 7. The PSL intensity was about the same as after the $504.5 \mathrm{~nm}$ bleach described above, i.e. very low. This means that the majority of the centers giving rise to the PSL and the tail in the excitation spectrum (cf figure 3 ) also give rise to a TL signal. Together with the conclusion of the preceding paragraph, this means that mainly, the centers giving rise to the PSL are identical to

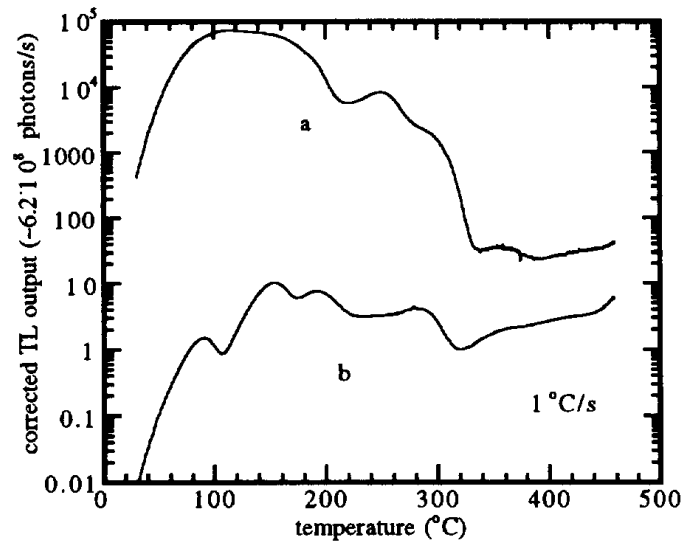

Figure 7. Corrected TL spectra for the unpolished sample from boule D. Curve a shows the TL spectrum measured after 8 minutes irradiation with the $\mathrm{Hg}$ lamp. Curve b shows the $\mathrm{TL}$ spectrum measured after 8 minutes irradiation with the $\mathrm{Hg}$ lamp, followed by a 59 minutes bleach using $504.5 \mathrm{~nm}$ light (accumulated surface dose $\sim 10^{19}$ photons $/ \mathrm{cm}^{2}$ ). The heating rate is $1^{\circ} \mathrm{C} / \mathrm{s}$.

the traps giving rise to the TL. Our results on the samples from boules $\mathrm{B}$ and $\mathrm{C}$ are similar, so this conclusion holds for all of the boules investigated.

\section{The relation between $T L$ and light output}

For the five unpolished samples, we measured the light output under ${ }^{137} \mathrm{Cs}$ gamma irradiation (662 keV), and we determined the integral of the TL curves between room temperature and $460^{\circ} \mathrm{C}$. The result is shown in figure 8. One can clearly see the anti-correlation between the TL intensity and the light output.

We also did annealing experiments on our samples. The samples were annealed in a heating cycle starting with an initial heating at $3.3^{\circ} \mathrm{C} / \mathrm{min}$. from room temperature to 1550 ${ }^{\circ} \mathrm{C}$, followed by 5 hours constant temperature of $1550{ }^{\circ} \mathrm{C}$, after which the temperature was lowered to room temperature again at a rate $-3.3^{\circ} \mathrm{C} / \mathrm{min}$.. In the first annealing cycle the sample was surrounded by a $98 \% \mathrm{Ar} / 2 \% \mathrm{H}_{2}$ atmosphere. After measurements on these annealed samples, a similar annealing cycle was performed, but in a $99.5 \%$ pure $\mathrm{O}_{2}$ atmosphere.

After the first annealing experiment, in $\mathrm{Ar} / \mathrm{H}_{2}$ atmosphere, the absorption spectra did not show large differences to those measured before annealing. In contrast to this, the scintillation light output was much less than before annealing and the $\mathrm{TL}$ intensity was higher. This is indicated in figure 8. After the second annealing experiment, in $\mathrm{O}_{2}$ atmosphere, the absorption in the $<350 \mathrm{~nm}$ wavelength region was markedly increased. On the average, the scintillation light output was slightly higher after the second annealing than before the two annealing cycles and the TL intensity was slightly lower. No annealing cycle altered the light output ordering of the 


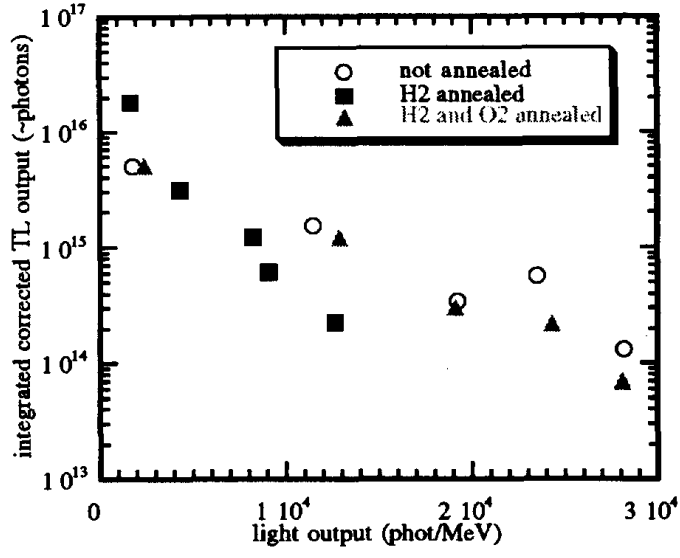

Figure 8. The light output and the integral of the corrected TL curve for the five unpolished samples. Before the TL measurement a 1 $\mathrm{mm}$ diameter circle of the samples was irradiated with the $\mathrm{Hg}$ lamp for 8 minutes (accumulated light dose through the circle: $-10^{13}$ photons). Shown are the results before annealing, after the $\mathrm{Ar} / \mathrm{H}_{2}$ anneal and after the additional $\mathrm{O}_{2}$ anneal. For each annealing stage, the data points pertain to the samples (from the left to the right): D, C, B, A(lower light output), A(highest light output).

samples: it remained $\mathrm{A}($ highest $)>\mathrm{A}($ lower $)>\mathrm{B}>\mathrm{C}>\mathrm{D}$.

\section{DISCUSSION AND CONCLUSIONS}

We give an explanation for the results presented in this paper. We note that the interpretation is preliminary: more research is necessary to support our interpretation.

First, we consider the absorption band at $276 \mathrm{~nm}$ in figure 2. The position and the width of this band are similar to those observed for $\mathrm{Ce}^{4+}$ and other 4+ charged rare earth ions in several oxide lattices $[5,6]$. The fact that extra absorption at wavelengths $<350 \mathrm{~nm}$ appears after annealing in an $\mathrm{O}_{2}$ atmosphere supports the assignment of the $276 \mathrm{~nm}$ absorption band to $\mathrm{Ce}^{4+}$. Then figures 2 and 5 tell us that if $\mathrm{Ce}^{3+}$ is excited, apparently $\mathrm{Ce}^{4+}$ can be created. This can be understood when realizing that, for instance, in $\mathrm{Y}_{3} \mathrm{Al}_{5} \mathrm{O}_{12}$ crystals [7], the $\mathrm{Ce}^{3+} 5 \mathrm{~d}$ levels are close to or higher than the bottom of the conduction band. This situation might be present in $\mathrm{Lu}_{2}\left(\mathrm{SiO}_{4}\right) \mathrm{O}$ as well. Then, if $\mathrm{Ce}^{3+}$ is excited into a $5 \mathrm{~d}$ state, the $5 \mathrm{~d}$ electron can enter the conduction band, leaving behind a $\mathrm{Ce}^{4+}$ ion.

Second, we consider the absorption tail in figure 2 , which is connected to the excitation tail in figure 3 , and which extends from $400 \mathrm{~nm}$ to beyond $700 \mathrm{~nm}$. The tail is probably not composed of many less broad absorption bands, each belonging to a particular center, because then we would expect bleaching at $504.5 \mathrm{~nm}$ to result in some kind of hole burning. Instead, no change was observed in the shape of the tail when bleaching with $504.5 \mathrm{~nm}$ light. We have shown that the tail is connected to PSL as well as to TL. Hence, the tail is ascribed to the emptying of traps, maybe involving transitions from trap levels below the conduction band to energy levels in the conduction band. Glow curve analysis shows that the traps observed in the TL spectra in figure 6 have a depth between 1.0 and $1.7 \mathrm{eV}$, so photons with a wavelength $<1200 \mathrm{~nm}$ may be able to empty the traps, which is consistent with the wavelength position of the tail in the absorption spectrum.

Finally, we address the relation between the TL intensity, i.e. the concentration of traps, and the scintillation light output. The mere presence of traps in a crystal is not a reason in itself for expecting a low scintillation light output. As soon as the traps are filled, the light output may be high again. But we never observed any variation of the light output of our crystals as a function of the concentration of filled traps. This means that a low light output is not caused by the traps, but by non-radiative recombination centers accompanying them (except of course if the traps are also non-radiative recombination centers). In this view, both traps and nonradiative recombination centers would have a common cause, e.g. disorder in the lattice. We note that after annealing in $\mathrm{Ar} / \mathrm{H}_{2}$ atmosphere, the light output was less than before annealing and after annealing in $\mathrm{O}_{2}$ atmosphere it was slightly higher. This suggests that oxygen vacancies may be involved in the reduction of the light output.

\section{ACKNOWLEDGEMENTS}

We are grateful to R.A. Manente for growing the crystals. R.A. Manente and C.A. Peterson are acknowledged for useful discussions on the subject of this paper.

\section{REFERENCES}

[1] C.L. Melcher and J.S. Schweitzer, IEEE Trans.Nucl.Sci. 39(4): 502-505 (1992)

[2] H. Suzuki, T.A. Tombrello, C.L. Melcher and J.S. Schweitzer, Nucl.Instr.Meth. A320: 263-272 (1992)

[3] P. Dorenbos, C.W.E. van Eijk, A.J.J. Bos and C.L. Melcher, to be published in the proceedings of the ICL'93 conference, Storrs, Connecticut, USA: J.Luminesc. (1993)

[4] H. Suzuki, T.A. Tombrello, C.L. Melcher and J.S. Schweitzer, IEEE Trans.Nucl.Sci. NS-40(4): 380-383 (1993)

[5] H.E. Hoefdraad, J.Inorg.Nucl.Chem. 37(9): 1917-1921 (1975)

[6] N. van Vugt, T. Wigmans and G. Blasse, J.Inorg.Nucl.Chem. 35: $2601-2602$ (1973)

[7] D.S. Hamilton, S.K. Gayen, G.J. Pogatshnik, R.D. Ghen and W.J. Miniscalco, Phys.Rev. B 39(13): 8807-8815 (1989) 